Supporting Information for:

\title{
21-Telluraporphyrins. 3. Synthesis, Structure, and Spectral Properties of a 21,21-Dihalo-21-Telluraporphyrin
}

Masako Abe, Michael R. Detty,* Oksana O. Gerlits, and Dinesh K. Sukumaran 
Table S1. Atomic Fractional Coordinates and Isotropic Thermal Parameters for compound 7 at $90 \mathrm{~K}$.

\begin{tabular}{|c|c|c|c|c|}
\hline Atom & $x$ & Y & $z$ & $\mathrm{U}(\mathrm{eq})$ \\
\hline $\operatorname{Te}(1)$ & $3581(1)$ & $879(1)$ & $14575(1)$ & $20(1)$ \\
\hline$O(1)$ & $3090(1)$ & $-6036(2)$ & $16782(2)$ & $26(1)$ \\
\hline$O(2)$ & $4992(1)$ & $1547(2)$ & $20770(1)$ & $28(1)$ \\
\hline $\mathrm{N}(1)$ & $3268(1)$ & $-1000(2)$ & $14399(2)$ & $19(1)$ \\
\hline$N(2)$ & $3831(1)$ & $-405(2)$ & $16250(2)$ & $16(1)$ \\
\hline $\mathrm{N}(3)$ & $4075(1)$ & $1858(2)$ & $15932(2)$ & $20(1)$ \\
\hline $\mathrm{C}(1)$ & $3703(1)$ & $2359(3)$ & $14216(2)$ & $17(1)$ \\
\hline C (2) & $3471(1)$ & $2482(3)$ & $13335(2)$ & $19(1)$ \\
\hline C (3) & $3223(1)$ & $1646(3)$ & $12884(2)$ & $18(1)$ \\
\hline C ( 4) & $3217(1)$ & $672(3)$ & $13309(2)$ & $20(1)$ \\
\hline$C(5)$ & $3019(1)$ & $-304(3)$ & $12999(2)$ & $18(1)$ \\
\hline$C(6)$ & $3058(1)$ & $-1155(3)$ & $13570(2)$ & $18(1)$ \\
\hline$C(7)$ & $2906(1)$ & $-2251(3)$ & $13396(2)$ & $20(1)$ \\
\hline$C(8)$ & $3023(1)$ & $-2739(3)$ & $14137(2)$ & $20(1)$ \\
\hline C (9) & $3257(1)$ & $-1948(3)$ & $14778(2)$ & $18(1)$ \\
\hline$C(10)$ & 3455 (1) & $-2146(3)$ & $15631(2)$ & $17(1)$ \\
\hline C (11) & $3726(1)$ & $-1467(3)$ & $16291(2)$ & $18(1)$ \\
\hline C (12) & $3937(1)$ & $-1766(3)$ & $17138(2)$ & $20(1)$ \\
\hline C (13) & $4155(1)$ & $-908(3)$ & $17591(2)$ & $19(1)$ \\
\hline C (14) & $4086(1)$ & $-25(3)$ & $17041(2)$ & $17(1)$ \\
\hline$C(15)$ & 4255 (1) & $1019(3)$ & $17290(2)$ & $18(1)$ \\
\hline$C(16)$ & $4254(1)$ & $1881(3)$ & $16781(2)$ & $17(1)$ \\
\hline C (17) & $4451(1)$ & $2920(3)$ & $17064(2)$ & $19(1)$ \\
\hline C (18) & $4392(1)$ & $3488(3)$ & $16374(2)$ & $20(1)$ \\
\hline C (19) & $4145(1)$ & 2815 (3) & $15669(2)$ & $18(1)$ \\
\hline$C(20)$ & $3971(1)$ & $3074(3)$ & $14801(2)$ & $17(1)$ \\
\hline$C(21)$ & $2799(1)$ & $-510(3)$ & $12098(2)$ & $19(1)$ \\
\hline C (22) & $2993(1)$ & $-268(3)$ & $11593(2)$ & $20(1)$ \\
\hline C (23) & $2805(1)$ & $-532(3)$ & $10759(2)$ & $25(1)$ \\
\hline C (24) & $2426(1)$ & $-1062(3)$ & $10411(2)$ & $30(1)$ \\
\hline$C(25)$ & $2222(1)$ & $-1285(3)$ & $10895(2)$ & $33(1)$ \\
\hline$C(26)$ & $2404(1)$ & $-1000(3)$ & $11728(2)$ & $27(1)$ \\
\hline$C(27)$ & $3368(1)$ & $-3198(3)$ & $15918(2)$ & $17(1)$ \\
\hline $\mathrm{C}(28)$ & $3652(1)$ & $-4028(3)$ & $16170(3)$ & $37(1)$ \\
\hline C (29) & $3572(1)$ & $-4985(3)$ & $16465(3)$ & $35(1)$ \\
\hline C (30) & $3202(1)$ & $-5117(3)$ & $16518(2)$ & $21(1)$ \\
\hline C (31) & $2914(1)$ & $-4291(3)$ & $16276(2)$ & $22(1)$ \\
\hline C (32) & $2995(1)$ & $-3344(3)$ & $15977(2)$ & $18(1)$ \\
\hline C (33) & $3355(1)$ & $-6941(3)$ & $16931(2)$ & $36(1)$ \\
\hline$C(34)$ & 4447 (1) & $1194(2)$ & $18207(2)$ & $16(1)$ \\
\hline$C(35)$ & $4197(1)$ & $1140(3)$ & $18623(2)$ & $19(1)$ \\
\hline$C(36)$ & $4364(1)$ & $1257(3)$ & $19469(2)$ & $20(1)$ \\
\hline C (37) & $4792(1)$ & $1414(3)$ & $19928(2)$ & $19(1)$ \\
\hline C (38) & $5048(1)$ & $1456(3)$ & $19532(2)$ & $20(1)$ \\
\hline C (39) & $4879(1)$ & $1348(3)$ & $18683(2)$ & $19(1)$ \\
\hline$C(40)$ & $4737(1)$ & $1469(4)$ & $21198(2)$ & $46(1)$ \\
\hline C ( 41$)$ & $4093(1)$ & 4107 (3) & $14540(2)$ & $18(1)$ \\
\hline$C(44)$ & $4319(1)$ & $5990(3)$ & $14021(2)$ & $28(1)$ \\
\hline C ( 42) & $3940(3)$ & $5068(7)$ & $14650(5)$ & $22(2)$ \\
\hline$C(43)$ & $4053(3)$ & $6002(8)$ & $14404(5)$ & $28(2)$ \\
\hline$C(45)$ & $4428(3)$ & $5009(6)$ & $13820(5)$ & $31(2)$ \\
\hline
\end{tabular}




\begin{tabular}{lllll}
$C(46)$ & $4318(2)$ & $4052(5)$ & $14083(5)$ & $25(1)$ \\
$C(47)$ & $3824(3)$ & $4975(8)$ & $14249(5)$ & $20(2)$ \\
$C(48)$ & $3947(4)$ & $5904(9)$ & $14002(6)$ & $27(2)$ \\
$C(49)$ & $4622(3)$ & $5164(7)$ & $14374(6)$ & $33(2)$ \\
$C(50)$ & $4512(3)$ & $4247(7)$ & $14648(6)$ & $31(2)$ \\
\hline
\end{tabular}


Table S2. Anisotropic displacement parameters $\left(\AA^{2} \times 10^{3}\right)$ for compound 7 at $90 \mathrm{~K}$.

\begin{tabular}{|c|c|c|c|c|c|c|}
\hline Atom & U11 & U22 & U33 & $\mathrm{U} 23$ & U13 & $\mathrm{U} 12$ \\
\hline$\overline{\mathrm{Te}}(1)$ & $26(1)$ & $19(1)$ & $15(1)$ & $4(1)$ & $8(1)$ & $-2(1)$ \\
\hline$O(1)$ & $34(1)$ & $19(1)$ & $30(1)$ & $4(1)$ & $19(1)$ & $-3(1)$ \\
\hline$O(2)$ & $23(1)$ & $50(2)$ & $14(1)$ & $2(1)$ & $11(1)$ & $1(1)$ \\
\hline $\mathrm{N}(1)$ & $19(1)$ & $20(2)$ & $21(1)$ & $5(1)$ & $11(1)$ & $0(1)$ \\
\hline$N(2)$ & $19(1)$ & $16(1)$ & $19(1)$ & $-1(1)$ & $12(1)$ & $-1(1)$ \\
\hline $\mathrm{N}(3)$ & $28(2)$ & $18(1)$ & $18(1)$ & $2(1)$ & $15(1)$ & $1(1)$ \\
\hline$C(1)$ & $22(2)$ & $16(2)$ & $17(2)$ & $2(1)$ & $13(1)$ & $2(1)$ \\
\hline C (2) & $24(2)$ & $19(2)$ & $19(2)$ & $3(1)$ & $14(2)$ & $3(1)$ \\
\hline C (3) & $18(2)$ & $22(2)$ & $14(2)$ & $7(1)$ & $9(1)$ & $6(1)$ \\
\hline C (4) & $14(2)$ & $31(2)$ & $17(2)$ & $7(1)$ & $8(1)$ & $4(1)$ \\
\hline C (5) & $14(2)$ & $25(2)$ & $18(2)$ & $3(1)$ & $10(1)$ & $2(1)$ \\
\hline$C(6)$ & $14(2)$ & $23(2)$ & $20(2)$ & $4(1)$ & $9(1)$ & $2(1)$ \\
\hline C (7) & $19(2)$ & $24(2)$ & $19(2)$ & $1(1)$ & $9(1)$ & $-1(1)$ \\
\hline C (8) & $20(2)$ & $19(2)$ & $24(2)$ & $2(1)$ & $13(2)$ & $0(1)$ \\
\hline C (9) & $17(2)$ & $20(2)$ & $20(2)$ & $5(1)$ & $11(1)$ & $0(1)$ \\
\hline$C(10)$ & $16(2)$ & $18(2)$ & $22(2)$ & $5(1)$ & $12(1)$ & $3(1)$ \\
\hline C (11) & $20(2)$ & $17(2)$ & $22(2)$ & $2(1)$ & $14(1)$ & $3(1)$ \\
\hline$C(12)$ & $24(2)$ & $18(2)$ & $20(2)$ & $5(1)$ & $12(2)$ & $3(1)$ \\
\hline C (13) & $19(2)$ & $22(2)$ & $17(2)$ & $5(1)$ & $10(1)$ & $4(2)$ \\
\hline C (14) & $19(2)$ & $19(2)$ & $16(2)$ & $2(1)$ & $11(1)$ & $3(1)$ \\
\hline C (15) & $17(2)$ & $21(2)$ & $16(2)$ & $1(1)$ & $9(1)$ & $3(1)$ \\
\hline$C(16)$ & $21(2)$ & $18(2)$ & $16(2)$ & $0(1)$ & $11(1)$ & $3(1)$ \\
\hline C (17) & $23(2)$ & $20(2)$ & $16(2)$ & $-3(1)$ & $10(1)$ & $2(1)$ \\
\hline C (18) & $25(2)$ & $17(2)$ & $20(2)$ & $1(1)$ & $13(2)$ & $2(1)$ \\
\hline C (19) & $26(2)$ & $15(2)$ & $18(2)$ & $2(1)$ & $15(2)$ & $3(1)$ \\
\hline$C(20)$ & $19(2)$ & $16(2)$ & $21(2)$ & $4(1)$ & $13(1)$ & $5(1)$ \\
\hline C (21) & $17(2)$ & $20(2)$ & $17(2)$ & $4(1)$ & $6(1)$ & $1(1)$ \\
\hline C (22) & $17(2)$ & $24(2)$ & $19(2)$ & $4(1)$ & $8(1)$ & $-2(1)$ \\
\hline$C(23)$ & $25(2)$ & $30(2)$ & $21(2)$ & $1(2)$ & $12(2)$ & $-3(2)$ \\
\hline C (24) & $32(2)$ & $35(2)$ & $16(2)$ & $0(2)$ & $4(2)$ & $-10(2)$ \\
\hline C (25) & $23(2)$ & $39(2)$ & $25(2)$ & $6(2)$ & $2(2)$ & $-13(2)$ \\
\hline$C(26)$ & $18(2)$ & $35(2)$ & $26(2)$ & $8(2)$ & $8(2)$ & $-4(2)$ \\
\hline C (27) & $19(2)$ & $17(2)$ & $14(2)$ & $3(1)$ & $7(1)$ & $-1(1)$ \\
\hline C (28) & $21(2)$ & $27(2)$ & $72(3)$ & $19(2)$ & $30(2)$ & $6(2)$ \\
\hline C (29) & $22(2)$ & $24(2)$ & $62(3)$ & $19(2)$ & $22(2)$ & $11(2)$ \\
\hline$C(30)$ & $26(2)$ & $19(2)$ & $17(2)$ & $2(1)$ & $11(2)$ & $-3(1)$ \\
\hline C (31) & $23(2)$ & $24(2)$ & $26(2)$ & $-4(1)$ & $19(2)$ & $-3(1)$ \\
\hline C (32) & $19(2)$ & $17(2)$ & $20(2)$ & $-3(1)$ & $11(1)$ & $1(1)$ \\
\hline C (33) & $43(2)$ & $21(2)$ & $42(2)$ & $11(2)$ & $19(2)$ & $-3(2)$ \\
\hline C (34) & $22(2)$ & $15(2)$ & $15(2)$ & $3(1)$ & $11(1)$ & $0(1)$ \\
\hline C (35) & $15(2)$ & $22(2)$ & $21(2)$ & $-1(1)$ & $10(1)$ & $-1(1)$ \\
\hline C (36) & $23(2)$ & $24(2)$ & $23(2)$ & $2(1)$ & $18(2)$ & $-1(1)$ \\
\hline C (37) & $25(2)$ & $20(2)$ & $14(2)$ & $4(1)$ & $10(1)$ & $2(1)$ \\
\hline C (38) & $18(2)$ & $26(2)$ & $18(2)$ & $4(1)$ & $10(1)$ & $0(1)$ \\
\hline C (39) & $22(2)$ & $21(2)$ & $21(2)$ & $4(1)$ & $16(2)$ & $2(1)$ \\
\hline$C(40)$ & $31(2)$ & $95(4)$ & $16(2)$ & $2(2)$ & $14(2)$ & $0(2)$ \\
\hline C (41) & $25(2)$ & $18(2)$ & $15(2)$ & $3(1)$ & $12(1)$ & $-2(2)$ \\
\hline$C(44)$ & $41(2)$ & $20(2)$ & $27(2)$ & $6(2)$ & $20(2)$ & $-3(2)$ \\
\hline$C(42)$ & $25(5)$ & $22(4)$ & $24(4)$ & $2(4)$ & $16(4)$ & $-1(3)$ \\
\hline C (43) & $38(5)$ & $17(4)$ & $29(5)$ & $3(5)$ & $15(5)$ & $6(4)$ \\
\hline C (45) & $47(5)$ & $30(4)$ & $30(4)$ & $2(3)$ & $31(4)$ & $-1(4)$ \\
\hline
\end{tabular}




\begin{tabular}{llllrrr}
$C(46)$ & $37(4)$ & $13(3)$ & $34(4)$ & $-1(3)$ & $25(4)$ & $-4(3)$ \\
$\mathrm{C}(47)$ & $19(5)$ & $27(5)$ & $21(5)$ & $3(5)$ & $14(5)$ & $3(4)$ \\
$\mathrm{C}(48)$ & $41(7)$ & $15(5)$ & $31(5)$ & $17(5)$ & $23(6)$ & $12(5)$ \\
$\mathrm{C}(49)$ & $23(4)$ & $34(5)$ & $42(6)$ & $13(4)$ & $15(4)$ & $-6(4)$ \\
$\mathrm{C}(50)$ & $20(4)$ & $22(5)$ & $46(6)$ & $11(4)$ & $10(4)$ & $4(3)$ \\
\hline
\end{tabular}


Table S3. Selected Bond Lengths $(\AA)$ and Angles $\left({ }^{\circ}\right)$ for compound 7 at $90 \mathrm{~K}$.

\begin{tabular}{|c|c|c|c|}
\hline $\operatorname{Te}(1)-C(1)$ & $2.066(3)$ & $\mathrm{C}(18)-\mathrm{C}(19)$ & $1.447(4)$ \\
\hline $\mathrm{Te}(1)-\mathrm{C}(4)$ & $2.075(3)$ & $\mathrm{C}(19)-\mathrm{C}(20)$ & $1.439(4)$ \\
\hline $\mathrm{Te}(1)-\mathrm{N}(1)$ & $2.558(3)$ & $\mathrm{C}(20)-\mathrm{C}(41)$ & $1.503(4)$ \\
\hline $\mathrm{O}(1)-\mathrm{C}(30)$ & $1.368(4)$ & $\mathrm{C}(21)-\mathrm{C}(22)$ & $1.399(5)$ \\
\hline $\mathrm{O}(1)-\mathrm{C}(33)$ & $1.421(4)$ & $C(21)-C(26)$ & $1.401(5)$ \\
\hline $\mathrm{O}(2)-\mathrm{C}(37)$ & $1.368(4)$ & $\mathrm{C}(22)-\mathrm{C}(23)$ & $1.385(5)$ \\
\hline $\mathrm{O}(2)-\mathrm{C}(40)$ & $1.435(4)$ & $C(23)-C(24)$ & $1.380(5)$ \\
\hline $\mathrm{N}(1)-\mathrm{C}(6)$ & $1.354(4)$ & $C(24)-C(25)$ & $1.389(5)$ \\
\hline $\mathrm{N}(1)-\mathrm{C}(9)$ & $1.375(4)$ & $C(25)-C(26)$ & $1.389(5)$ \\
\hline $\mathrm{N}(2)-\mathrm{C}(14)$ & $1.389(4)$ & $\mathrm{C}(27)-\mathrm{C}(28)$ & $1.376(5)$ \\
\hline $\mathrm{N}(2)-\mathrm{C}(11)$ & $1.389(4)$ & $\mathrm{C}(27)-\mathrm{C}(32)$ & $1.394(4)$ \\
\hline N(3)-C(19) & $1.348(4)$ & $C(28)-C(29)$ & $1.388(5)$ \\
\hline $\mathrm{N}(3)-\mathrm{C}(16)$ & $1.368(4)$ & $C(29)-C(30)$ & $1.374(5)$ \\
\hline$C(1)-C(20)$ & $1.386(5)$ & $C(30)-C(31)$ & $1.382(5)$ \\
\hline$C(1)-C(2)$ & $1.434(4)$ & $\mathrm{C}(31)-\mathrm{C}(32)$ & $1.382(4)$ \\
\hline$C(2)-C(3)$ & $1.375(5)$ & $C(34)-C(35)$ & $1.397(4)$ \\
\hline$C(3)-C(4)$ & $1.443(4)$ & $\mathrm{C}(34)-\mathrm{C}(39)$ & $1.404(5)$ \\
\hline$C(4)-C(5)$ & $1.394(5)$ & $C(35)-C(36)$ & $1.375(4)$ \\
\hline$C(5)-C(6)$ & $1.442(4)$ & $\mathrm{C}(36)-\mathrm{C}(37)$ & $1.387(5)$ \\
\hline$C(5)-C(21)$ & $1.476(4)$ & $\mathrm{C}(37)-\mathrm{C}(38)$ & $1.390(5)$ \\
\hline$C(6)-C(7)$ & $1.454(5)$ & $\mathrm{C}(38)-\mathrm{C}(39)$ & $1.377(4)$ \\
\hline$C(7)-C(8)$ & $1.353(4)$ & $\mathrm{C}(41)-\mathrm{C}(42)$ & $1.37(1)$ \\
\hline $\mathrm{C}(8)-\mathrm{C}(9)$ & $1.465(5)$ & $\mathrm{C}(41)-\mathrm{C}(47)$ & $1.39(1)$ \\
\hline$C(9)-C(10)$ & $1.397(4)$ & $\mathrm{C}(41)-\mathrm{C}(46)$ & $1.386(7)$ \\
\hline $\mathrm{C}(10)-\mathrm{C}(11)$ & $1.429(5)$ & $\mathrm{C}(41)-\mathrm{C}(50)$ & $1.431(9)$ \\
\hline $\mathrm{C}(10)-\mathrm{C}(27)$ & $1.493(4)$ & $\mathrm{C}(44)-\mathrm{C}(48)$ & $1.32(1)$ \\
\hline $\mathrm{C}(11)-\mathrm{C}(12)$ & $1.417(4)$ & $\mathrm{C}(44)-\mathrm{C}(45)$ & $1.382(8)$ \\
\hline C(12)-C(13) & $1.360(5)$ & $\mathrm{C}(44)-\mathrm{C}(43)$ & $1.40(1)$ \\
\hline $\mathrm{C}(13)-\mathrm{C}(14)$ & $1.428(4)$ & $\mathrm{C}(44)-\mathrm{C}(49)$ & $1.422(9)$ \\
\hline$C(14)-C(15)$ & $1.422(4)$ & $\mathrm{C}(42)-\mathrm{C}(43)$ & $1.37(1)$ \\
\hline$C(15)-C(16)$ & $1.413(4)$ & $\mathrm{C}(45)-\mathrm{C}(46)$ & $1.404(9)$ \\
\hline$C(15)-C(34)$ & $1.495(4)$ & $\mathrm{C}(47)-\mathrm{C}(48)$ & $1.38(2)$ \\
\hline$C(16)-C(17)$ & $1.456(4)$ & $\mathrm{C}(49)-\mathrm{C}(50)$ & $1.37(1)$ \\
\hline$C(17)-C(18)$ & $1.363(4)$ & & \\
\hline $\mathrm{C}(1)-\mathrm{Te}(1)-\mathrm{C}(4)$ & 83.4(1) & $\mathrm{C}(4)-\mathrm{C}(5)-\mathrm{C}(6)$ & 119.2(3) \\
\hline $\mathrm{C}(1)-\mathrm{Te}(1)-\mathrm{N}(1)$ & $157.4(1)$ & $C(4)-C(5)-C(21)$ & 121.3(3) \\
\hline $\mathrm{C}(4)-\mathrm{Te}(1)-\mathrm{N}(1)$ & $74.0(1)$ & $C(6)-C(5)-C(21)$ & $119.4(3)$ \\
\hline $\mathrm{C}(30)-\mathrm{O}(1)-\mathrm{C}(33)$ & $117.4(3)$ & $\mathrm{N}(1)-\mathrm{C}(6)-\mathrm{C}(5)$ & $120.8(3)$ \\
\hline $\mathrm{C}(37)-\mathrm{O}(2)-\mathrm{C}(40)$ & $116.3(3)$ & $\mathrm{N}(1)-\mathrm{C}(6)-\mathrm{C}(7)$ & $110.0(3)$ \\
\hline $\mathrm{C}(6)-\mathrm{N}(1)-\mathrm{C}(9)$ & $107.5(3)$ & $\mathrm{C}(5)-\mathrm{C}(6)-\mathrm{C}(7)$ & $129.2(3)$ \\
\hline $\mathrm{C}(6)-\mathrm{N}(1)-\mathrm{Te}(1)$ & $105.2(2)$ & $\mathrm{C}(8)-\mathrm{C}(7)-\mathrm{C}(6)$ & $106.9(3)$ \\
\hline $\mathrm{C}(9)-\mathrm{N}(1)-\mathrm{Te}(1)$ & $146.7(2)$ & $\mathrm{C}(7)-\mathrm{C}(8)-\mathrm{C}(9)$ & 106.9(3) \\
\hline $\mathrm{C}(14)-\mathrm{N}(2)-\mathrm{C}(11)$ & 109.9(3) & $\mathrm{N}(1)-\mathrm{C}(9)-\mathrm{C}(10)$ & $125.8(3)$ \\
\hline $\mathrm{C}(19)-\mathrm{N}(3)-\mathrm{C}(16)$ & $107.9(3)$ & $\mathrm{N}(1)-\mathrm{C}(9)-\mathrm{C}(8)$ & 108.7(3) \\
\hline $\mathrm{C}(20)-\mathrm{C}(1)-\mathrm{C}(2)$ & $129.4(3)$ & $\mathrm{C}(10)-\mathrm{C}(9)-\mathrm{C}(8)$ & $125.4(3)$ \\
\hline $\mathrm{C}(20)-\mathrm{C}(1)-\mathrm{Te}(1)$ & $120.5(2)$ & $\mathrm{C}(9)-\mathrm{C}(10)-\mathrm{C}(11)$ & $129.2(3)$ \\
\hline $\mathrm{C}(2)-\mathrm{C}(1)-\mathrm{Te}(1)$ & $110.1(2)$ & $\mathrm{C}(9)-\mathrm{C}(10)-\mathrm{C}(27)$ & 117.1(3) \\
\hline $\mathrm{C}(3)-\mathrm{C}(2)-\mathrm{C}(1)$ & $118.3(3)$ & $\mathrm{C}(11)-\mathrm{C}(10)-\mathrm{C}(27)$ & $113.7(3)$ \\
\hline $\mathrm{C}(2)-\mathrm{C}(3)-\mathrm{C}(4)$ & $118.9(3)$ & $\mathrm{N}(2)-\mathrm{C}(11)-\mathrm{C}(12)$ & $106.4(3)$ \\
\hline$C(5)-C(4)-C(3)$ & 130.4(3) & $\mathrm{N}(2)-\mathrm{C}(11)-\mathrm{C}(10)$ & $128.3(3)$ \\
\hline $\mathrm{C}(5)-\mathrm{C}(4)-\mathrm{Te}(1)$ & $120.5(2)$ & $\mathrm{C}(12)-\mathrm{C}(11)-\mathrm{C}(10)$ & $125.3(3)$ \\
\hline $\mathrm{C}(3)-\mathrm{C}(4)-\mathrm{Te}(1)$ & 109.1(2) & $\mathrm{C}(13)-\mathrm{C}(12)-\mathrm{C}(11)$ & $109.0(3)$ \\
\hline
\end{tabular}




\begin{tabular}{|c|c|c|c|}
\hline$C(12)-C(13)-C(14)$ & $108.4(3)$ & $\mathrm{C}(29)-\mathrm{C}(30)-\mathrm{C}(31)$ & $119.5(3)$ \\
\hline $\mathrm{N}(2)-\mathrm{C}(14)-\mathrm{C}(15)$ & $128.9(3)$ & $\mathrm{C}(30)-\mathrm{C}(31)-\mathrm{C}(32)$ & $120.4(3)$ \\
\hline $\mathrm{N}(2)-\mathrm{C}(14)-\mathrm{C}(13)$ & $106.2(3)$ & $\mathrm{C}(31)-\mathrm{C}(32)-\mathrm{C}(27)$ & $120.9(3)$ \\
\hline$C(15)-C(14)-C(13)$ & $124.8(3)$ & $\mathrm{C}(35)-\mathrm{C}(34)-\mathrm{C}(39)$ & $117.9(3)$ \\
\hline$C(16)-C(15)-C(14)$ & $127.9(3)$ & $C(35)-C(34)-C(15)$ & $119.8(3)$ \\
\hline $\mathrm{C}(16)-\mathrm{C}(15)-\mathrm{C}(34)$ & $118.3(3)$ & $\mathrm{C}(39)-\mathrm{C}(34)-\mathrm{C}(15)$ & $122.2(3)$ \\
\hline $\mathrm{C}(14)-\mathrm{C}(15)-\mathrm{C}(34)$ & $113.8(3)$ & $\mathrm{C}(36)-\mathrm{C}(35)-\mathrm{C}(34)$ & $121.7(3)$ \\
\hline $\mathrm{N}(3)-\mathrm{C}(16)-\mathrm{C}(15)$ & $125.1(3)$ & $\mathrm{C}(35)-\mathrm{C}(36)-\mathrm{C}(37)$ & $119.5(3)$ \\
\hline N(3)-C(16)-C(17) & $108.7(3)$ & $\mathrm{O}(2)-\mathrm{C}(37)-\mathrm{C}(36)$ & $124.8(3)$ \\
\hline$C(15)-C(16)-C(17)$ & $126.2(3)$ & $\mathrm{O}(2)-\mathrm{C}(37)-\mathrm{C}(38)$ & $115.2(3)$ \\
\hline $\mathrm{C}(18)-\mathrm{C}(17)-\mathrm{C}(16)$ & $106.8(3)$ & $\mathrm{C}(36)-\mathrm{C}(37)-\mathrm{C}(38)$ & $120.0(3)$ \\
\hline $\mathrm{C}(17)-\mathrm{C}(18)-\mathrm{C}(19)$ & $106.5(3)$ & $\mathrm{C}(39)-\mathrm{C}(38)-\mathrm{C}(37)$ & $120.2(3)$ \\
\hline $\mathrm{N}(3)-\mathrm{C}(19)-\mathrm{C}(20)$ & $121.5(3)$ & $\mathrm{C}(38)-\mathrm{C}(39)-\mathrm{C}(34)$ & $120.6(3)$ \\
\hline $\mathrm{N}(3)-\mathrm{C}(19)-\mathrm{C}(18)$ & $110.0(3)$ & $\mathrm{C}(42)-\mathrm{C}(41)-\mathrm{C}(46)$ & $120.3(5)$ \\
\hline $\mathrm{C}(20)-\mathrm{C}(19)-\mathrm{C}(18)$ & $128.4(3)$ & $\mathrm{C}(47)-\mathrm{C}(41)-\mathrm{C}(46)$ & $107.4(5)$ \\
\hline$C(1)-C(20)-C(19)$ & $119.9(3)$ & $\mathrm{C}(42)-\mathrm{C}(41)-\mathrm{C}(50)$ & $109.5(6)$ \\
\hline $\mathrm{C}(1)-\mathrm{C}(20)-\mathrm{C}(41)$ & $120.9(3)$ & $C(47)-C(41)-C(50)$ & $117.4(6)$ \\
\hline $\mathrm{C}(19)-\mathrm{C}(20)-\mathrm{C}(41)$ & $119.2(3)$ & $\mathrm{C}(42)-\mathrm{C}(41)-\mathrm{C}(20)$ & 121.1(4) \\
\hline $\mathrm{C}(22)-\mathrm{C}(21)-\mathrm{C}(26)$ & $118.0(3)$ & $\mathrm{C}(47)-\mathrm{C}(41)-\mathrm{C}(20)$ & $122.9(5)$ \\
\hline$C(22)-C(21)-C(5)$ & $120.1(3)$ & $\mathrm{C}(46)-\mathrm{C}(41)-\mathrm{C}(20)$ & $118.0(4)$ \\
\hline $\mathrm{C}(26)-\mathrm{C}(21)-\mathrm{C}(5)$ & $121.8(3)$ & $\mathrm{C}(50)-\mathrm{C}(41)-\mathrm{C}(20)$ & $119.6(4)$ \\
\hline $\mathrm{C}(23)-\mathrm{C}(22)-\mathrm{C}(21)$ & $120.8(3)$ & $\mathrm{C}(48)-\mathrm{C}(44)-\mathrm{C}(45)$ & $108.7(7)$ \\
\hline $\mathrm{C}(24)-\mathrm{C}(23)-\mathrm{C}(22)$ & $120.7(3)$ & $\mathrm{C}(45)-\mathrm{C}(44)-\mathrm{C}(43)$ & $118.1(6)$ \\
\hline$C(23)-C(24)-C(25)$ & $119.4(3)$ & $\mathrm{C}(48)-\mathrm{C}(44)-\mathrm{C}(49)$ & $120.5(6)$ \\
\hline$C(24)-C(25)-C(26)$ & $120.2(3)$ & $\mathrm{C}(43)-\mathrm{C}(44)-\mathrm{C}(49)$ & $109.5(6)$ \\
\hline$C(25)-C(26)-C(21)$ & $120.8(3)$ & $\mathrm{C}(41)-\mathrm{C}(42)-\mathrm{C}(43)$ & $120.2(8)$ \\
\hline $\mathrm{C}(28)-\mathrm{C}(27)-\mathrm{C}(32)$ & $117.6(3)$ & $\mathrm{C}(42)-\mathrm{C}(43)-\mathrm{C}(44)$ & $120.9(9)$ \\
\hline $\mathrm{C}(28)-\mathrm{C}(27)-\mathrm{C}(10)$ & $122.8(3)$ & $\mathrm{C}(44)-\mathrm{C}(45)-\mathrm{C}(46)$ & $120.8(6)$ \\
\hline $\mathrm{C}(32)-\mathrm{C}(27)-\mathrm{C}(10)$ & $119.6(3)$ & $\mathrm{C}(41)-\mathrm{C}(46)-\mathrm{C}(45)$ & $118.8(6)$ \\
\hline $\mathrm{C}(27)-\mathrm{C}(28)-\mathrm{C}(29)$ & $122.0(3)$ & $\mathrm{C}(48)-\mathrm{C}(47)-\mathrm{C}(41)$ & $121.0(9)$ \\
\hline $\mathrm{C}(30)-\mathrm{C}(29)-\mathrm{C}(28)$ & $119.7(3)$ & $\mathrm{C}(44)-\mathrm{C}(48)-\mathrm{C}(47)$ & $121(1)$ \\
\hline $\mathrm{O}(1)-\mathrm{C}(30)-\mathrm{C}(29)$ & $124.2(3)$ & $\mathrm{C}(50)-\mathrm{C}(49)-\mathrm{C}(44)$ & $119.1(7)$ \\
\hline $\mathrm{O}(1)-\mathrm{C}(30)-\mathrm{C}(31)$ & $116.3(3)$ & $\mathrm{C}(49)-\mathrm{C}(50)-\mathrm{C}(41)$ & $120.1(7)$ \\
\hline
\end{tabular}


Table S4. Atomic Fractional Coordinates and Isotropic Thermal Parameters for compound $\mathbf{1 5}$ at $90 \mathrm{~K}$.

\begin{tabular}{|c|c|c|c|c|}
\hline Atom & $\mathrm{x}$ & $\mathrm{y}$ & z & $\mathrm{U}(\mathrm{eq})$ \\
\hline $\mathrm{Te}(1)$ & $2790(1)$ & $-8823(1)$ & $8143(1)$ & $16(1)$ \\
\hline $\mathrm{Cl}(1)$ & $3705(1)$ & $-7624(1)$ & $7600(1)$ & $21(1)$ \\
\hline $\mathrm{Cl}(2)$ & 2055 (1) & $-10221(1)$ & $8840(1)$ & $24(1)$ \\
\hline$O(1)$ & $552(1)$ & $-8691(2)$ & $3781(1)$ & $24(1)$ \\
\hline$O(2)$ & $543(1)$ & $-394(2)$ & $7711(1)$ & 21 (1) \\
\hline$N(1)$ & $2682(1)$ & $-9554(2)$ & 6971 (1) & $19(1)$ \\
\hline $\mathrm{N}(2)$ & $2115(1)$ & $-6858(2)$ & $7088(1)$ & $16(1)$ \\
\hline $\mathrm{N}(3)$ & $2621(1)$ & $-6526(2)$ & $8503(1)$ & $17(1)$ \\
\hline$C(1)$ & $3450(2)$ & $-8547(3)$ & $8965(1)$ & $19(1)$ \\
\hline$C(2)$ & $3883(2)$ & $-9617(3)$ & $9075(1)$ & $20(1)$ \\
\hline$C(3)$ & $3883(2)$ & $-10541(3)$ & $8625(1)$ & $19(1)$ \\
\hline$C(4)$ & $3452(2)$ & $-10397(3)$ & $8062(1)$ & $18(1)$ \\
\hline$C(5)$ & $3439(2)$ & $-11079(3)$ & $7517(1)$ & $17(1)$ \\
\hline$C(6)$ & $3039(2)$ & $-10636(3)$ & $6971(1)$ & $19(1)$ \\
\hline$C(7)$ & $2963(2)$ & $-11220(3)$ & $6360(2)$ & $23(1)$ \\
\hline$C(8)$ & $2556(2)$ & $-10446(3)$ & 5989 (1) & $22(1)$ \\
\hline$C(9)$ & $2373(2)$ & $-9393(3)$ & $6380(1)$ & $19(1)$ \\
\hline C (10) & $1941(2)$ & $-8394(3)$ & $6189(1)$ & $18(1)$ \\
\hline C (11) & $1771(2)$ & $-7327(3)$ & $6552(1)$ & $16(1)$ \\
\hline C (12) & $1162(2)$ & $-6584(3)$ & $6428(1)$ & $20(1)$ \\
\hline C (13) & $1151(2)$ & $-5670(3)$ & $6883(1)$ & $19(1)$ \\
\hline C (14) & $1758(2)$ & $-5833(3)$ & $7296(1)$ & $16(1)$ \\
\hline C (15) & $1909(2)$ & $-5067(3)$ & $7839(1)$ & $16(1)$ \\
\hline$C(16)$ & $2317(2)$ & $-5381(3)$ & $8380(1)$ & $17(1)$ \\
\hline C (17) & $2518(2)$ & $-4536(3)$ & $8895(1)$ & $19(1)$ \\
\hline C (18) & $2947(2)$ & $-5187(3)$ & $9306(1)$ & $21(1)$ \\
\hline C (19) & $3006(2)$ & $-6432(3)$ & $9049(1)$ & $18(1)$ \\
\hline C (20) & $3434(2)$ & $-7449(3)$ & $9291(1)$ & $20(1)$ \\
\hline C (21) & $3830(2)$ & $-12283(3)$ & $7472(1)$ & $19(1)$ \\
\hline C (22) & $3667(2)$ & $-13250(3)$ & $7869(2)$ & $23(1)$ \\
\hline C (23) & $3990(2)$ & $-14408(3)$ & $7814(2)$ & $28(1)$ \\
\hline C (24) & $4477(2)$ & $-14598(3)$ & $7358(2)$ & $29(1)$ \\
\hline C (25) & $4647(2)$ & $-13633(3)$ & $6969(2)$ & $28(1)$ \\
\hline$C(26)$ & $4326(2)$ & $-12472(3)$ & $7024(2)$ & $23(1)$ \\
\hline C (27) & $1579(2)$ & $-8466(3)$ & $5556(1)$ & $19(1)$ \\
\hline C (28) & $1164(2)$ & $-9491(3)$ & $5367(1)$ & $21(1)$ \\
\hline C (29) & $831(2)$ & $-9539(3)$ & $4773(1)$ & $21(1)$ \\
\hline C (30) & $905(2)$ & $-8562(3)$ & $4354(1)$ & $19(1)$ \\
\hline C (31) & $1312(2)$ & $-7523(3)$ & $4532(1)$ & $19(1)$ \\
\hline C (32) & $1640(2)$ & $-7484(3)$ & $5131(1)$ & $18(1)$ \\
\hline C (33) & $621(2)$ & $-7702(3)$ & $3344(2)$ & $27(1)$ \\
\hline C (34) & $1567(2)$ & $-3827(3)$ & $7807(1)$ & $16(1)$ \\
\hline C (35) & $1673(2)$ & $-3032(3)$ & $7304(1)$ & $18(1)$ \\
\hline$C(36)$ & $1352(2)$ & $-1869(3)$ & $7256(1)$ & $19(1)$ \\
\hline C (37) & $901(2)$ & $-1500(3)$ & $7716(1)$ & $17(1)$ \\
\hline C (38) & $788(2)$ & $-2277(3)$ & $8223(1)$ & $19(1)$ \\
\hline C (39) & $1121(2)$ & $-3422(3)$ & $8269(1)$ & $19(1)$ \\
\hline$C(40)$ & $673(2)$ & $447(3)$ & $7213(2)$ & $24(1)$ \\
\hline$C(41 A)$ & $3932(2)$ & $-7203(5)$ & $9841(2)$ & $20(2)$ \\
\hline$C(42)$ & $3643(2)$ & $-6926(5)$ & $10409(2)$ & $24(1)$ \\
\hline
\end{tabular}




\begin{tabular}{|c|c|c|c|c|}
\hline C ( 43) & $4086(3)$ & $-6696(5)$ & $10937(2)$ & $33(2)$ \\
\hline C ( 44) & $4819(2)$ & $-6742(6)$ & $10897(2)$ & $36(2)$ \\
\hline C (45) & $5108(2)$ & $-7019(5)$ & $10330(2)$ & $39(2)$ \\
\hline$C(46)$ & $4664(2)$ & $-7250(5)$ & $9801(2)$ & $26(1)$ \\
\hline$C(41 B)$ & $3833(3)$ & $-7331(5)$ & $9932(2)$ & $28(2)$ \\
\hline$C(47)$ & $4336(3)$ & $-6393(5)$ & $10021(2)$ & $37(2)$ \\
\hline C (48) & $4684(3)$ & $-6229(5)$ & $10605(3)$ & $47(2)$ \\
\hline C (49) & $4529(3)$ & $-7003(6)$ & $11101(2)$ & $47(3)$ \\
\hline$C(50)$ & $4026(3)$ & $-7941(6)$ & $11011(2)$ & $42(2)$ \\
\hline C (51) & $3679(3)$ & $-8105(5)$ & $10427(2)$ & $31(2)$ \\
\hline$O(1 S)$ & $252(4)$ & $-2316(8)$ & $9640(4)$ & $19(2)$ \\
\hline$C(2 S)$ & $75(7)$ & $-218(12)$ & $10080(6)$ & $21(2)$ \\
\hline$C(3 S)$ & $503(7)$ & $-1391(11)$ & $10058(6)$ & $28(3)$ \\
\hline$C(1 S)$ & $2622(3)$ & $-842(6)$ & $10247(3)$ & $56(1)$ \\
\hline$C l(1 A)$ & $1826(3)$ & $-1747(7)$ & $10306(4)$ & $56(1)$ \\
\hline $\mathrm{Cl}(2 \mathrm{~A})$ & $2816(4)$ & $-301(7)$ & $11019(3)$ & $56(1)$ \\
\hline $\mathrm{Cl}(3 \mathrm{~A})$ & $3252(3)$ & $-2004(6)$ & $10032(4)$ & $56(1)$ \\
\hline $\mathrm{Cl}(1 \mathrm{~B})$ & $1776(3)$ & $-1350(7)$ & $10475(3)$ & $56(1)$ \\
\hline $\mathrm{Cl}(2 \mathrm{~B})$ & $3096(4)$ & $-115(7)$ & $10902(3)$ & $56(1)$ \\
\hline $\mathrm{Cl}(3 \mathrm{~B})$ & $3077(4)$ & $-2154(6)$ & $9982(4)$ & $56(1)$ \\
\hline
\end{tabular}


Table S5. Anisotropic displacement parameters $\left(\AA^{2} \times 10^{3}\right)$ for compound 15 at $90 \mathrm{~K}$.

\begin{tabular}{|c|c|c|c|c|c|c|}
\hline Atom & U11 & U22 & U33 & U23 & U13 & U12 \\
\hline $\mathrm{Te}(1)$ & $16(1)$ & $16(1)$ & $15(1)$ & $-2(1)$ & $-1(1)$ & $2(1)$ \\
\hline $\mathrm{Cl}(1)$ & $19(1)$ & $19(1)$ & $23(1)$ & $0(1)$ & $2(1)$ & $1(1)$ \\
\hline $\mathrm{Cl}$ (2) & $22(1)$ & $23(1)$ & $28(1)$ & $-2(1)$ & $4(1)$ & $-4(1)$ \\
\hline$O(1)$ & $26(1)$ & $26(1)$ & $18(1)$ & $-1(1)$ & $-5(1)$ & $-4(1)$ \\
\hline$O(2)$ & $20(1)$ & $16(1)$ & $26(1)$ & $1(1)$ & $2(1)$ & $5(1)$ \\
\hline $\mathrm{N}(1)$ & $23(1)$ & $16(1)$ & $17(1)$ & $0(1)$ & $-1(1)$ & $2(1)$ \\
\hline $\mathrm{N}(2)$ & $18(1)$ & $16(1)$ & $14(1)$ & $-1(1)$ & $-1(1)$ & $3(1)$ \\
\hline N (3) & $18(1)$ & $17(1)$ & $16(1)$ & $-2(1)$ & $-1(1)$ & $3(1)$ \\
\hline$C(1)$ & $18(1)$ & $22(1)$ & $17(1)$ & $0(1)$ & $-3(1)$ & $-1(1)$ \\
\hline$C(2)$ & $19(1)$ & $21(1)$ & $19(1)$ & $1(1)$ & $-3(1)$ & $1(1)$ \\
\hline C (3) & $18(1)$ & $17(1)$ & $22(1)$ & $2(1)$ & $-1(1)$ & $2(1)$ \\
\hline$C(4)$ & $18(1)$ & $14(1)$ & $22(1)$ & $0(1)$ & $1(1)$ & $2(1)$ \\
\hline C (5) & $16(1)$ & $16(1)$ & $20(1)$ & $2(1)$ & $2(1)$ & $1(1)$ \\
\hline$C(6)$ & $22(1)$ & $18(1)$ & $17(1)$ & $0(1)$ & $2(1)$ & $2(1)$ \\
\hline$C(7)$ & $28(2)$ & $21(1)$ & $20(1)$ & $-2(1)$ & $2(1)$ & $6(1)$ \\
\hline$C(8)$ & $31(2)$ & $20(1)$ & $15(1)$ & $-3(1)$ & $3(1)$ & $4(1)$ \\
\hline C (9) & $24(1)$ & $18(1)$ & $15(1)$ & $0(1)$ & $1(1)$ & $2(1)$ \\
\hline$C(10)$ & $21(1)$ & $16(1)$ & $15(1)$ & $-1(1)$ & $2(1)$ & $0(1)$ \\
\hline C (11) & $19(1)$ & $16(1)$ & $14(1)$ & $0(1)$ & $1(1)$ & $0(1)$ \\
\hline $\mathrm{C}(12)$ & $20(1)$ & $22(1)$ & $17(1)$ & $-1(1)$ & $-2(1)$ & $1(1)$ \\
\hline C (13) & $18(1)$ & $19(1)$ & $19(1)$ & $1(1)$ & $-1(1)$ & $3(1)$ \\
\hline C (14) & $18(1)$ & $14(1)$ & $15(1)$ & $0(1)$ & $1(1)$ & $1(1)$ \\
\hline C (15) & $16(1)$ & $15(1)$ & $17(1)$ & $-1(1)$ & $1(1)$ & $1(1)$ \\
\hline$C(16)$ & $18(1)$ & $15(1)$ & $18(1)$ & $-3(1)$ & $1(1)$ & $3(1)$ \\
\hline C (17) & $21(1)$ & $19(1)$ & $18(1)$ & $-5(1)$ & $0(1)$ & $2(1)$ \\
\hline$C(18)$ & $23(1)$ & $21(1)$ & $18(1)$ & $-5(1)$ & $0(1)$ & $3(1)$ \\
\hline C (19) & $16(1)$ & $21(1)$ & $16(1)$ & $-2(1)$ & $0(1)$ & $3(1)$ \\
\hline$C(20)$ & $17(1)$ & $22(1)$ & $20(1)$ & $-1(1)$ & $-1(1)$ & $2(1)$ \\
\hline$C(21)$ & $17(1)$ & $18(1)$ & $22(1)$ & $-2(1)$ & $1(1)$ & $1(1)$ \\
\hline C (22) & $26(2)$ & $16(1)$ & $27(2)$ & $-3(1)$ & $2(1)$ & $0(1)$ \\
\hline C (23) & $35(2)$ & $17(1)$ & $33(2)$ & -1 (1) & $-3(1)$ & $-1(1)$ \\
\hline C (24) & $26(2)$ & $17(1)$ & $41(2)$ & $-9(1)$ & $-4(1)$ & $5(1)$ \\
\hline C (25) & $20(1)$ & $28(2)$ & $36(2)$ & $-11(1)$ & $3(1)$ & $5(1)$ \\
\hline$C(26)$ & $21(1)$ & $21(1)$ & $27(2)$ & $-1(1)$ & $4(1)$ & $2(1)$ \\
\hline$C(27)$ & $18(1)$ & $27(1)$ & $11(1)$ & $-4(1)$ & $-1(1)$ & $-3(1)$ \\
\hline C (28) & $28(2)$ & $19(1)$ & $16(1)$ & $0(1)$ & $4(1)$ & $-1(1)$ \\
\hline$C(29)$ & $25(1)$ & $20(1)$ & $19(1)$ & $-4(1)$ & $3(1)$ & $-5(1)$ \\
\hline$C(30)$ & $19(1)$ & $24(1)$ & $15(1)$ & $-3(1)$ & $1(1)$ & $0(1)$ \\
\hline C (31) & $22(1)$ & $19(1)$ & $16(1)$ & $1(1)$ & $1(1)$ & $1(1)$ \\
\hline C (32) & $24(1)$ & $15(1)$ & $16(1)$ & $-1(1)$ & $0(1)$ & $0(1)$ \\
\hline C (33) & $29(2)$ & $32(2)$ & $18(1)$ & $2(1)$ & $-5(1)$ & $-1(1)$ \\
\hline C (34) & $17(1)$ & $14(1)$ & $16(1)$ & $-1(1)$ & $0(1)$ & $2(1)$ \\
\hline C ( 35$)$ & $20(1)$ & $17(1)$ & $17(1)$ & $-1(1)$ & $4(1)$ & $2(1)$ \\
\hline$C(36)$ & $21(1)$ & $16(1)$ & $20(1)$ & $0(1)$ & $0(1)$ & $1(1)$ \\
\hline C (37) & $13(1)$ & $15(1)$ & $22(1)$ & $-1(1)$ & $-1(1)$ & $1(1)$ \\
\hline C (38) & $18(1)$ & $19(1)$ & $20(1)$ & $-4(1)$ & $3(1)$ & $1(1)$ \\
\hline C (39) & $21(1)$ & $16(1)$ & $19(1)$ & $0(1)$ & $2(1)$ & $1(1)$ \\
\hline C (40) & $29(2)$ & $18(1)$ & $26(2)$ & $1(1)$ & $-1(1)$ & $5(1)$ \\
\hline$C(41 A)$ & $31(4)$ & $15(3)$ & $13(3)$ & $0(3)$ & $-4(3)$ & $7(3)$ \\
\hline$C(42)$ & $27(3)$ & $27(3)$ & $17(3)$ & $0(2)$ & $-1(2)$ & $3(2)$ \\
\hline$C(43)$ & $51(5)$ & $32(4)$ & $17(3)$ & $-2(3)$ & $-11(3)$ & $12(3)$ \\
\hline
\end{tabular}




\begin{tabular}{llllrrr}
$C(44)$ & $39(5)$ & $36(4)$ & $31(4)$ & $-9(4)$ & $-23(4)$ & $14(4)$ \\
$C(45)$ & $24(3)$ & $45(5)$ & $45(4)$ & $-7(4)$ & $-16(3)$ & $7(3)$ \\
$C(46)$ & $18(3)$ & $29(3)$ & $30(3)$ & $-9(3)$ & $-1(2)$ & $0(2)$ \\
$C(41 B)$ & $22(4)$ & $28(5)$ & $32(4)$ & $-9(4)$ & $-10(3)$ & $4(3)$ \\
$C(47)$ & $33(4)$ & $36(4)$ & $40(4)$ & $-1(3)$ & $-13(3)$ & $-5(3)$ \\
$C(48)$ & $46(5)$ & $48(5)$ & $44(5)$ & $-15(4)$ & $-22(4)$ & $-7(4)$ \\
$C(49)$ & $39(5)$ & $69(7)$ & $32(5)$ & $-18(5)$ & $-11(4)$ & $13(5)$ \\
$C(50)$ & $41(4)$ & $63(6)$ & $20(3)$ & $-5(4)$ & $-11(3)$ & $13(4)$ \\
$C(51)$ & $29(3)$ & $42(4)$ & $22(3)$ & $-6(3)$ & $-3(3)$ & $6(3)$ \\
\hline
\end{tabular}


Table S6. Selected Bond Lengths $(\AA)$ and Angles $\left(^{\circ}\right)$ for compound 15 at $90 \mathrm{~K}$.

\begin{tabular}{|c|c|c|c|}
\hline $\mathrm{Te}(1)-\mathrm{C}(4)$ & $2.117(3)$ & $\mathrm{C}(21)-\mathrm{C}(22)$ & $1.388(4)$ \\
\hline $\mathrm{Te}(1)-\mathrm{C}(1)$ & $2.118(3)$ & $C(21)-C(26)$ & $1.389(4)$ \\
\hline $\mathrm{Te}(1)-\mathrm{Cl}(1)$ & $2.4918(8)$ & $\mathrm{C}(22)-\mathrm{C}(23)$ & $1.394(5)$ \\
\hline $\mathrm{Te}(1)-\mathrm{Cl}(2)$ & $2.5751(8)$ & $\mathrm{C}(23)-\mathrm{C}(24)$ & $1.391(5)$ \\
\hline $\mathrm{O}(1)-\mathrm{C}(30)$ & $1.368(4)$ & $\mathrm{C}(24)-\mathrm{C}(25)$ & $1.379(5)$ \\
\hline $\mathrm{O}(1)-\mathrm{C}(33)$ & $1.428(4)$ & $C(25)-C(26)$ & $1.395(5)$ \\
\hline $\mathrm{O}(2)-\mathrm{C}(37)$ & $1.366(3)$ & $\mathrm{C}(27)-\mathrm{C}(28)$ & $1.399(4)$ \\
\hline $\mathrm{O}(2)-\mathrm{C}(40)$ & $1.428(4)$ & $\mathrm{C}(27)-\mathrm{C}(32)$ & $1.402(4)$ \\
\hline $\mathrm{N}(1)-\mathrm{C}(6)$ & $1.344(4)$ & $\mathrm{C}(28)-\mathrm{C}(29)$ & $1.385(4)$ \\
\hline $\mathrm{N}(1)-\mathrm{C}(9)$ & $1.372(4)$ & $C(29)-C(30)$ & $1.391(4)$ \\
\hline $\mathrm{N}(2)-\mathrm{C}(14)$ & $1.378(4)$ & $\mathrm{C}(30)-\mathrm{C}(31)$ & $1.396(4)$ \\
\hline $\mathrm{N}(2)-\mathrm{C}(11)$ & $1.380(4)$ & $\mathrm{C}(31)-\mathrm{C}(32)$ & $1.393(4)$ \\
\hline $\mathrm{N}(3)-\mathrm{C}(19)$ & $1.342(4)$ & $C(34)-C(35)$ & $1.397(4)$ \\
\hline $\mathrm{N}(3)-\mathrm{C}(16)$ & $1.378(4)$ & $\mathrm{C}(34)-\mathrm{C}(39)$ & $1.401(4)$ \\
\hline $\mathrm{C}(1)-\mathrm{C}(20)$ & $1.373(4)$ & $\mathrm{C}(35)-\mathrm{C}(36)$ & $1.392(4)$ \\
\hline$C(1)-C(2)$ & $1.423(4)$ & $\mathrm{C}(36)-\mathrm{C}(37)$ & $1.393(4)$ \\
\hline $\mathrm{C}(2)-\mathrm{C}(3)$ & $1.381(4)$ & $\mathrm{C}(37)-\mathrm{C}(38)$ & $1.394(4)$ \\
\hline $\mathrm{C}(3)-\mathrm{C}(4)$ & $1.423(4)$ & $\mathrm{C}(38)-\mathrm{C}(39)$ & $1.383(4)$ \\
\hline $\mathrm{C}(4)-\mathrm{C}(5)$ & $1.375(4)$ & $\mathrm{C}(41 \mathrm{~A})-\mathrm{C}(42)$ & 1.3900 \\
\hline$C(5)-C(6)$ & $1.435(4)$ & $C(41 A)-C(46)$ & 1.3900 \\
\hline$C(5)-C(21)$ & $1.496(4)$ & $C(42)-C(43)$ & 1.3900 \\
\hline$C(6)-C(7)$ & $1.450(4)$ & $\mathrm{C}(43)-\mathrm{C}(44)$ & 1.3900 \\
\hline $\mathrm{C}(7)-\mathrm{C}(8)$ & $1.358(4)$ & $\mathrm{C}(44)-\mathrm{C}(45)$ & 1.3900 \\
\hline $\mathrm{C}(8)-\mathrm{C}(9)$ & $1.459(4)$ & $\mathrm{C}(45)-\mathrm{C}(46)$ & 1.3900 \\
\hline $\mathrm{C}(9)-\mathrm{C}(10)$ & $1.397(4)$ & $\mathrm{C}(41 \mathrm{~B})-\mathrm{C}(47)$ & 1.3900 \\
\hline $\mathrm{C}(10)-\mathrm{C}(11)$ & $1.432(4)$ & $\mathrm{C}(41 \mathrm{~B})-\mathrm{C}(51)$ & 1.3900 \\
\hline $\mathrm{C}(10)-\mathrm{C}(27)$ & $1.483(4)$ & $\mathrm{C}(47)-\mathrm{C}(48)$ & 1.3900 \\
\hline$C(11)-C(12)$ & $1.411(4)$ & $\mathrm{C}(48)-\mathrm{C}(49)$ & 1.3900 \\
\hline$C(12)-C(13)$ & $1.383(4)$ & $\mathrm{C}(49)-\mathrm{C}(50)$ & 1.3900 \\
\hline $\mathrm{C}(13)-\mathrm{C}(14)$ & $1.418(4)$ & $\mathrm{C}(50)-\mathrm{C}(51)$ & 1.3900 \\
\hline $\mathrm{C}(14)-\mathrm{C}(15)$ & $1.440(4)$ & $\mathrm{O}(1 \mathrm{~S})-\mathrm{C}(3 \mathrm{~S})$ & $1.403(5)$ \\
\hline$C(15)-C(16)$ & $1.393(4)$ & $\mathrm{C}(2 \mathrm{~S})-\mathrm{C}(3 \mathrm{~S})$ & $1.50(2)$ \\
\hline$C(15)-C(34)$ & $1.482(4)$ & $\mathrm{C}(1 \mathrm{~S})-\mathrm{Cl}(3 \mathrm{~B})$ & $1.761(5)$ \\
\hline $\mathrm{C}(16)-\mathrm{C}(17)$ & $1.461(4)$ & $\mathrm{C}(1 \mathrm{~S})-\mathrm{Cl}(2 \mathrm{~A})$ & $1.769(5)$ \\
\hline$C(17)-C(18)$ & $1.355(4)$ & $\mathrm{C}(1 \mathrm{~S})-\mathrm{Cl}(1 \mathrm{~B})$ & $1.779(5)$ \\
\hline$C(18)-C(19)$ & $1.454(4)$ & $\mathrm{C}(1 \mathrm{~S})-\mathrm{Cl}(2 \mathrm{~B})$ & $1.798(5)$ \\
\hline C(19)-C(20) & $1.439(4)$ & $\mathrm{C}(1 \mathrm{~S})-\mathrm{Cl}(3 \mathrm{~A})$ & $1.800(5)$ \\
\hline $\mathrm{C}(20)-\mathrm{C}(41 \mathrm{~A})$ & $1.487(4)$ & $\mathrm{C}(1 \mathrm{~S})-\mathrm{Cl}(1 \mathrm{~A})$ & $1.800(5)$ \\
\hline $\mathrm{C}(20)-\mathrm{C}(41 \mathrm{~B})$ & $1.530(5)$ & & \\
\hline $\mathrm{C}(4)-\mathrm{Te}(1)-\mathrm{C}(1)$ & $81.8(1)$ & $\mathrm{C}(2)-\mathrm{C}(1)-\mathrm{Te}(1)$ & $109.2(2)$ \\
\hline $\mathrm{C}(4)-\mathrm{Te}(1)-\mathrm{Cl}(1)$ & $87.03(8)$ & $\mathrm{C}(3)-\mathrm{C}(2)-\mathrm{C}(1)$ & $119.3(3)$ \\
\hline $\mathrm{C}(1)-\mathrm{Te}(1)-\mathrm{Cl}(1)$ & $85.62(9)$ & $\mathrm{C}(2)-\mathrm{C}(3)-\mathrm{C}(4)$ & $119.2(3)$ \\
\hline $\mathrm{C}(4)-\mathrm{Te}(1)-\mathrm{Cl}(2)$ & $85.39(8)$ & $\mathrm{C}(5)-\mathrm{C}(4)-\mathrm{C}(3)$ & $129.9(3)$ \\
\hline $\mathrm{C}(1)-\mathrm{Te}(1)-\mathrm{Cl}(2)$ & $84.95(9)$ & $\mathrm{C}(5)-\mathrm{C}(4)-\mathrm{Te}(1)$ & $120.8(2)$ \\
\hline $\mathrm{Cl}(1)-\mathrm{Te}(1)-\mathrm{Cl}(2)$ & $168.65(3)$ & $\mathrm{C}(3)-\mathrm{C}(4)-\mathrm{Te}(1)$ & $109.3(2)$ \\
\hline $\mathrm{C}(30)-\mathrm{O}(1)-\mathrm{C}(33)$ & $117.0(3)$ & $\mathrm{C}(4)-\mathrm{C}(5)-\mathrm{C}(6)$ & $119.7(3)$ \\
\hline $\mathrm{C}(37)-\mathrm{O}(2)-\mathrm{C}(40)$ & $116.9(2)$ & $\mathrm{C}(4)-\mathrm{C}(5)-\mathrm{C}(21)$ & $122.0(3)$ \\
\hline $\mathrm{C}(6)-\mathrm{N}(1)-\mathrm{C}(9)$ & $107.3(3)$ & $\mathrm{C}(6)-\mathrm{C}(5)-\mathrm{C}(21)$ & $118.2(3)$ \\
\hline $\mathrm{C}(14)-\mathrm{N}(2)-\mathrm{C}(11)$ & $110.0(2)$ & $\mathrm{N}(1)-\mathrm{C}(6)-\mathrm{C}(5)$ & $121.9(3)$ \\
\hline $\mathrm{C}(19)-\mathrm{N}(3)-\mathrm{C}(16)$ & $107.4(2)$ & $\mathrm{N}(1)-\mathrm{C}(6)-\mathrm{C}(7)$ & $110.4(3)$ \\
\hline $\mathrm{C}(20)-\mathrm{C}(1)-\mathrm{C}(2)$ & $129.8(3)$ & $\mathrm{C}(5)-\mathrm{C}(6)-\mathrm{C}(7)$ & $127.6(3)$ \\
\hline $\mathrm{C}(20)-\mathrm{C}(1)-\mathrm{Te}(1)$ & $120.9(2)$ & $\mathrm{C}(8)-\mathrm{C}(7)-\mathrm{C}(6)$ & $106.5(3)$ \\
\hline
\end{tabular}




\begin{tabular}{|c|c|c|c|}
\hline $\mathrm{C}(7)-\mathrm{C}(8)-\mathrm{C}(9)$ & $106.6(3)$ & $\mathrm{C}(32)-\mathrm{C}(27)-\mathrm{C}(10)$ & $120.1(3)$ \\
\hline $\mathrm{N}(1)-\mathrm{C}(9)-\mathrm{C}(10)$ & $125.3(3)$ & $\mathrm{C}(29)-\mathrm{C}(28)-\mathrm{C}(27)$ & $120.8(3)$ \\
\hline $\mathrm{N}(1)-\mathrm{C}(9)-\mathrm{C}(8)$ & 109.1(3) & $\mathrm{C}(28)-\mathrm{C}(29)-\mathrm{C}(30)$ & $120.3(3)$ \\
\hline $\mathrm{C}(10)-\mathrm{C}(9)-\mathrm{C}(8)$ & $125.6(3)$ & $\mathrm{O}(1)-\mathrm{C}(30)-\mathrm{C}(29)$ & $116.0(3)$ \\
\hline $\mathrm{C}(9)-\mathrm{C}(10)-\mathrm{C}(11)$ & 127.1(3) & $\mathrm{O}(1)-\mathrm{C}(30)-\mathrm{C}(31)$ & $123.8(3)$ \\
\hline C(9)-C(10)-C(27) & $117.3(3)$ & $\mathrm{C}(29)-\mathrm{C}(30)-\mathrm{C}(31)$ & $120.2(3)$ \\
\hline $\mathrm{C}(11)-\mathrm{C}(10)-\mathrm{C}(27)$ & $115.5(3)$ & $\mathrm{C}(32)-\mathrm{C}(31)-\mathrm{C}(30)$ & $118.9(3)$ \\
\hline $\mathrm{N}(2)-\mathrm{C}(11)-\mathrm{C}(12)$ & $106.8(2)$ & $\mathrm{C}(31)-\mathrm{C}(32)-\mathrm{C}(27)$ & $121.6(3)$ \\
\hline $\mathrm{N}(2)-\mathrm{C}(11)-\mathrm{C}(10)$ & $129.3(3)$ & $C(35)-C(34)-C(39)$ & 118.1(3) \\
\hline $\mathrm{C}(12)-\mathrm{C}(11)-\mathrm{C}(10)$ & $123.8(3)$ & $C(35)-C(34)-C(15)$ & $120.3(3)$ \\
\hline $\mathrm{C}(13)-\mathrm{C}(12)-\mathrm{C}(11)$ & 108.3(3) & $\mathrm{C}(39)-\mathrm{C}(34)-\mathrm{C}(15)$ & $121.6(3)$ \\
\hline $\mathrm{C}(12)-\mathrm{C}(13)-\mathrm{C}(14)$ & $107.8(3)$ & $\mathrm{C}(36)-\mathrm{C}(35)-\mathrm{C}(34)$ & $121.7(3)$ \\
\hline $\mathrm{N}(2)-\mathrm{C}(14)-\mathrm{C}(13)$ & $106.9(2)$ & $\mathrm{C}(35)-\mathrm{C}(36)-\mathrm{C}(37)$ & $119.0(3)$ \\
\hline $\mathrm{N}(2)-\mathrm{C}(14)-\mathrm{C}(15)$ & $129.6(3)$ & $\mathrm{O}(2)-\mathrm{C}(37)-\mathrm{C}(36)$ & $124.2(3)$ \\
\hline $\mathrm{C}(13)-\mathrm{C}(14)-\mathrm{C}(15)$ & $123.3(3)$ & $\mathrm{O}(2)-\mathrm{C}(37)-\mathrm{C}(38)$ & $115.6(3)$ \\
\hline $\mathrm{C}(16)-\mathrm{C}(15)-\mathrm{C}(14)$ & $127.4(3)$ & $\mathrm{C}(36)-\mathrm{C}(37)-\mathrm{C}(38)$ & $120.2(3)$ \\
\hline $\mathrm{C}(16)-\mathrm{C}(15)-\mathrm{C}(34)$ & $118.4(3)$ & $\mathrm{C}(39)-\mathrm{C}(38)-\mathrm{C}(37)$ & $120.1(3)$ \\
\hline$C(14)-C(15)-C(34)$ & $114.2(2)$ & C(38)-C(39)-C(34) & $120.9(3)$ \\
\hline $\mathrm{N}(3)-\mathrm{C}(16)-\mathrm{C}(15)$ & $125.5(3)$ & $C(42)-C(41 A)-C(46)$ & 120.0 \\
\hline N(3)-C(16)-C(17) & $108.8(2)$ & $\mathrm{C}(42)-\mathrm{C}(41 \mathrm{~A})-\mathrm{C}(20)$ & $117.8(3)$ \\
\hline$C(15)-C(16)-C(17)$ & $125.7(3)$ & $\mathrm{C}(46)-\mathrm{C}(41 \mathrm{~A})-\mathrm{C}(20)$ & $122.2(3)$ \\
\hline $\mathrm{C}(18)-\mathrm{C}(17)-\mathrm{C}(16)$ & 106.7(3) & $\mathrm{C}(43)-\mathrm{C}(42)-\mathrm{C}(41 \mathrm{~A})$ & 120.0 \\
\hline $\mathrm{C}(17)-\mathrm{C}(18)-\mathrm{C}(19)$ & $106.6(3)$ & $\mathrm{C}(42)-\mathrm{C}(43)-\mathrm{C}(44)$ & 120.0 \\
\hline $\mathrm{N}(3)-\mathrm{C}(19)-\mathrm{C}(20)$ & $121.5(3)$ & $\mathrm{C}(45)-\mathrm{C}(44)-\mathrm{C}(43)$ & 120.0 \\
\hline N(3)-C(19)-C(18) & $110.4(3)$ & $\mathrm{C}(44)-\mathrm{C}(45)-\mathrm{C}(46)$ & 120.0 \\
\hline $\mathrm{C}(20)-\mathrm{C}(19)-\mathrm{C}(18)$ & $128.0(3)$ & $\mathrm{C}(45)-\mathrm{C}(46)-\mathrm{C}(41 \mathrm{~A})$ & 120.0 \\
\hline $\mathrm{C}(1)-\mathrm{C}(20)-\mathrm{C}(19)$ & $119.9(3)$ & $\mathrm{C}(47)-\mathrm{C}(41 \mathrm{~B})-\mathrm{C}(51)$ & 120.0 \\
\hline $\mathrm{C}(1)-\mathrm{C}(20)-\mathrm{C}(41 \mathrm{~A})$ & $121.6(3)$ & $\mathrm{C}(47)-\mathrm{C}(41 \mathrm{~B})-\mathrm{C}(20)$ & $118.8(3)$ \\
\hline$C(19)-C(20)-C(41 A)$ & $117.9(3)$ & $\mathrm{C}(51)-\mathrm{C}(41 \mathrm{~B})-\mathrm{C}(20)$ & $121.2(3)$ \\
\hline $\mathrm{C}(1)-\mathrm{C}(20)-\mathrm{C}(41 \mathrm{~B})$ & 120.1(3) & $\mathrm{C}(48)-\mathrm{C}(47)-\mathrm{C}(41 \mathrm{~B})$ & 120.0 \\
\hline $\mathrm{C}(19)-\mathrm{C}(20)-\mathrm{C}(41 \mathrm{~B})$ & $119.9(3)$ & $\mathrm{C}(47)-\mathrm{C}(48)-\mathrm{C}(49)$ & 120.0 \\
\hline $\mathrm{C}(22)-\mathrm{C}(21)-\mathrm{C}(26)$ & $119.5(3)$ & $\mathrm{C}(50)-\mathrm{C}(49)-\mathrm{C}(48)$ & 120.0 \\
\hline$C(22)-C(21)-C(5)$ & $118.8(3)$ & C(51)-C(50)-C(49) & 120.0 \\
\hline $\mathrm{C}(26)-\mathrm{C}(21)-\mathrm{C}(5)$ & $121.6(3)$ & $\mathrm{C}(50)-\mathrm{C}(51)-\mathrm{C}(41 \mathrm{~B})$ & 120.0 \\
\hline $\mathrm{C}(21)-\mathrm{C}(22)-\mathrm{C}(23)$ & $120.4(3)$ & $\mathrm{O}(1 \mathrm{~S})-\mathrm{C}(3 \mathrm{~S})-\mathrm{C}(2 \mathrm{~S})$ & $117(1)$ \\
\hline $\mathrm{C}(24)-\mathrm{C}(23)-\mathrm{C}(22)$ & $119.9(3)$ & $\mathrm{Cl}(3 \mathrm{~B})-\mathrm{C}(1 \mathrm{~S})-\mathrm{Cl}(1 \mathrm{~B})$ & $107.6(5)$ \\
\hline$C(25)-C(24)-C(23)$ & $119.8(3)$ & $\mathrm{Cl}(3 \mathrm{~B})-\mathrm{C}(1 \mathrm{~S})-\mathrm{Cl}(2 \mathrm{~B})$ & $111.7(5)$ \\
\hline$C(24)-C(25)-C(26)$ & $120.4(3)$ & $\mathrm{Cl}(1 \mathrm{~B})-\mathrm{C}(1 \mathrm{~S})-\mathrm{Cl}(2 \mathrm{~B})$ & $109.5(5)$ \\
\hline $\mathrm{C}(21)-\mathrm{C}(26)-\mathrm{C}(25)$ & $120.0(3)$ & $\mathrm{Cl}(2 \mathrm{~A})-\mathrm{C}(1 \mathrm{~S})-\mathrm{Cl}(3 \mathrm{~A})$ & $111.2(5)$ \\
\hline $\mathrm{C}(28)-\mathrm{C}(27)-\mathrm{C}(32)$ & 118.1(3) & $\mathrm{Cl}(2 \mathrm{~A})-\mathrm{C}(1 \mathrm{~S})-\mathrm{Cl}(1 \mathrm{~A})$ & $104.0(5)$ \\
\hline $\mathrm{C}(28)-\mathrm{C}(27)-\mathrm{C}(10)$ & $121.8(3)$ & $\mathrm{Cl}(3 \mathrm{~A})-\mathrm{C}(1 \mathrm{~S})-\mathrm{Cl}(1 \mathrm{~A})$ & $102.0(4)$ \\
\hline
\end{tabular}

